This item was submitted to Loughborough's Research Repository by the author.

Items in Figshare are protected by copyright, with all rights reserved, unless otherwise indicated.

\title{
Note on backward uniqueness for a class of parabolic equations
}

PLEASE CITE THE PUBLISHED VERSION

http://dx.doi.org/10.1007/978-3-319-48812-7_63

\section{PUBLISHER}

(c) Birkhauser Verlag

VERSION

AM (Accepted Manuscript)

\section{PUBLISHER STATEMENT}

This work is made available according to the conditions of the Creative Commons Attribution-NonCommercialNoDerivatives 4.0 International (CC BY-NC-ND 4.0) licence. Full details of this licence are available at: https://creativecommons.org/licenses/by-nc-nd/4.0/

\section{LICENCE}

CC BY-NC-ND 4.0

\section{REPOSITORY RECORD}

Jaeh, Christian. 2019. "Note on Backward Uniqueness for a Class of Parabolic Equations". figshare. https://hdl.handle.net/2134/24106. 


\title{
Note on backward uniqueness for a class of parabolic equations
}

\author{
Christian P. Jäh
}

\begin{abstract}
In this note, we review some recent results on the backward uniqueness for solutions of parabolic equations of second and higher order. The main focus is the connection of the backward uniqueness property with the regularity of the principal part coefficients measured by moduli of continuity. We announce a new backward uniqueness result for higher order equations.
\end{abstract}

Mathematics Subject Classification (2010). Primary 35Bxx, 35Kxx; Secondary 35K25, 35K30.

Keywords. Carleman estimates, backward uniqueness, parabolic equations, higher order equations, Bony's paraproduct, rough coefficients.

\section{Introduction}

We consider parabolic equations of the type

$$
P u=\partial_{t} u+\sum_{0 \leq|\alpha|,|\beta| \leq m}(-1)^{|\alpha|} \partial_{x}^{\alpha}\left(a_{\alpha \beta}(t, x) \partial_{x}^{\beta} u\right)=0
$$

on the strip $[0, T] \times \mathbb{R}_{x}^{n}$ with $m \in \mathbb{N}$. The $\alpha$ and $\beta$ are $n$-multiindices. We assume $a_{\alpha \beta}(t, x)=\overline{a_{\beta \alpha}(t, x)}$ for all $0 \leq|\alpha|,|\beta| \leq m$ and the $a_{\beta \alpha}$ are supposed to be real for $|\alpha|=|\beta|=m$ on $[0, T] \times \mathbb{R}_{x}^{n}$. We assume that there exists a $\kappa \in(0,1]$ such that $\sum_{|\alpha|=|\beta|=m} a_{\alpha \beta}(t, x) \xi^{\alpha} \xi^{\beta} \geq \kappa|\xi|^{2 m}$ for all $(t, x, \xi) \in$ $[0, T] \times \mathbb{R}_{x}^{n} \times \mathbb{R}_{\xi}^{n}$.

By saying that $P$ has the backward uniqueness property, we mean the following: Given $u \in \mathcal{H}, P u=0$ on $[0, T] \times \mathbb{R}_{x}^{n}$ with $u(T, x)=0$ on $\mathbb{R}_{x}^{n}$, then it follows that $u=0$ on $[0, T] \times \mathbb{R}_{x}^{n}$. The space $\mathcal{H}$ is an appropriate function space for the problem at hand. Here, we shall prove the backward uniqueness properties with respect to the space

$$
\mathcal{H}^{m}:=H^{1}\left([0, T], L^{2}\left(\mathbb{R}_{x}^{n}\right)\right) \cap L^{2}\left([0, T], H^{2 m}\left(\mathbb{R}_{x}^{n}\right)\right) .
$$

In [11], Lions and Malgrange proved backward uniqueness for $P$ in $\mathcal{H}^{m}$ under the condition that the $a_{\alpha \beta}$ are Lipschitz continuous with respect to 
$t$ and sufficiently smooth with respect to $x$. The latter requirement is to make the operator fall into the abstract framework in which the authors were working. In that paper, the authors also raised the question whether or not the Lipschitz regularity with respect to $t$ is really necessary or could be replaced by simple continuity. In [1], Bardos and Tatar proved essentially the same result as Lions and Malgrange replacing Lipschitz continuity with absolute continuity. In [10], Miller showed that a certain amount of regularity with respect to $t$ is necessary for the backward uniqueness property to hold in the case $m=1$. He constructed a counterexample with $\frac{1}{6}$-Hölder continuous principal part coefficients.

For $m=1$, Del Santo and Prizzi proved in [3] the backward uniqueness property for $P$ assuming the so-called Osgood condition for the modulus of continuity with respect to $t$. More precisely, let $\mu:[0,1] \rightarrow[0,1]$ be a modulus of continuity, i.e. a continuous, concave, and increasing function with $\mu(0)=0$. If the principal part coefficients belong to $C^{\mu}\left([0, T], L^{\infty}\left(\mathbb{R}_{x}^{n}\right)\right) \cap$ $L^{\infty}\left([0, T], B^{2}\left(\mathbb{R}_{x}^{n}\right)\right)$, then uniqueness holds in $\mathcal{H}^{2}$ if $\mu$ satisfies the Osgood condition

$$
\int_{0}^{1} \frac{d s}{\mu(s)}=+\infty .
$$

The high regularity with respect to $x$ was due to a difficult commutator estimate arising from the use of the Littlewood-Paley decomposition in the proof of a Carleman estimate needed for the uniqueness proof. This was overcome in [5], where the authors assumed that the principal part coefficients belong to the space $C^{\mu}\left([0, T], L^{\infty}\left(\mathbb{R}_{x}^{n}\right)\right) \cap L^{\infty}\left([0, T], \operatorname{Lip}\left(\mathbb{R}_{x}^{n}\right)\right)$. The Carleman estimate proved in [5] is on the level of $H^{-s}, s \in(0,1)$ instead of the usual $L^{2}$. The precise statement is

Proposition 1.1 (Prop. $3.1[5])$. Let $s \in(0,1)$ and $\mu$ be a modulus of continuity satisfying (1.2). Assume further that, for all $j, k=1, \ldots, n$,

$$
a_{j k} \in C^{\mu}\left([0, T], L^{\infty}\left(\mathbb{R}_{x}^{n}\right)\right) \cap L^{\infty}\left([0, T], \operatorname{Lip}\left(\mathbb{R}_{x}^{n}\right)\right) .
$$

Then there exists a strictly increasing $C^{2}$-function $\Phi:[0,+\infty) \rightarrow[0,+\infty)$ such that there exists a $\gamma_{0} \geq 1$ such that

$$
\begin{array}{r}
\int_{0}^{T / 2} e^{\frac{2}{\gamma} \Phi(\gamma(T-t))}\left\|\partial_{t} u+\sum_{j, k=1}^{n} \partial_{x_{j}}\left(a_{j k}(t, \cdot) \partial_{x_{k}} u\right)\right\|_{H^{-s}}^{2} d t \\
\quad \gtrsim \gamma^{\frac{1}{2}} \int_{0}^{T / 2} e^{\frac{2}{\gamma} \Phi(\gamma(T-t))}\left(\left\|\nabla_{x} u\right\|_{H^{-s}}^{2}+\gamma^{\frac{1}{2}}\|u\|_{H^{-s}}^{2}\right) d t
\end{array}
$$

for all $u \in C_{0}^{\infty}\left(\mathbb{R}_{t} \times \mathbb{R}_{x}^{n}\right)$ with $\operatorname{supp}(u) \subseteq[0, T / 2] \times \mathbb{R}_{x}^{n}$ and all $\gamma \geq \gamma_{0}$.

Remark 1.2. The weight function $\Phi$ is coupled to the modulus of continuity as follows. Let $\mu$ be the Osgood modulus of continuity. Then set

$$
\begin{aligned}
\eta(t) & :=\int_{\frac{1}{t}}^{1} \frac{1}{\mu(s)} d s, \quad t \geq 1 \\
\Phi(\tau) & :=\int_{0}^{\tau} \eta^{-1}(t) d t, \quad \tau \geq 0 .
\end{aligned}
$$


The function $\Phi$ is differentiable and, thanks to the Osgood condition, defined on $[0,+\infty)$. It also satisfies the nonlinear ordinary differential equation $\Phi^{\prime \prime}=\mu\left(1 / \Phi^{\prime}\right)\left(\Phi^{\prime}\right)^{2}$. This connection was first described in [14] for a Carleman estimate for a uniqueness result for the solutions of the Cauchy problem for second order elliptic equations with non-Lipschitz coefficients.

Remark 1.3. Replacing Proposition 3.5 in [5] by Theorem 2.5.8 in [12], one can recover the $L^{2}$ estimates, i.e. Proposition 1.1 holds true for $s=0$. This allows us to treat second order equations with all lower order terms with coefficients merely in $L^{\infty}\left([0, T] \times \mathbb{R}_{x}^{n}\right)$ not only terms of zero order as in [5]. See also Remark 2.4 in [5].

Finally, in [6], the authors proved a uniqueness result assuming that the principal part coefficients belong to $C^{\mu}\left([0, T], L^{\infty}\left(\mathbb{R}_{x}^{n}\right)\right) \cap L^{\infty}\left([0, T], C^{\omega}\left(\mathbb{R}_{x}^{n}\right)\right)$, where $\mu$ and $\omega$ are moduli of continuity, $\mu$ satisfies (1.2), and $\omega$ is given by $\omega(s)=\sqrt{\mu\left(s^{2}\right)}$. Unless in the case $\omega(s)=s$, the Carleman estimate will be at the level of a Sobolev space of negative order.

Proposition 1.4 (Prop. 7 in [6]). Let $\mu$ and $\omega$ be two moduli of continuity such that $\omega(s)=\sqrt{\mu\left(s^{2}\right)}$. Suppose that $\mu$ satisfies the Osgood condition (1.2). Suppose moreover that there exists a positive constant $C$ such that $\int_{0}^{h} \frac{\omega(t)}{t} d t \leq C \omega(h), \frac{\omega\left(2^{-q}\right)}{\omega\left(2^{-p}\right)} \leq C \omega\left(2^{p-q}\right)$ for $1 \leq p \leq q-1$, and, for all $s \in(0,1), \sum_{k=0}^{+\infty} 2^{(1-s) k} \omega\left(2^{-k}\right)<+\infty$. Assume further that, for all $j, k=1, \ldots, n$,

$$
a_{j k} \in C^{\mu}\left([0, T], L^{\infty}\left(\mathbb{R}_{x}^{n}\right)\right) \cap L^{\infty}\left([0, T], C^{\omega}\left(\mathbb{R}_{x}^{n}\right)\right) .
$$

Let $s \in(0,1)$. Then there exists a strictly increasing $C^{2}$-function $\Phi:[0,+\infty) \rightarrow$ $[0,+\infty)$ such that there exists a $\gamma_{0} \geq 1$ such that

$$
\begin{gathered}
\int_{0}^{T / 2} e^{\frac{2}{\gamma} \Phi(\gamma(T-t))}\left\|\partial_{t} u+\sum_{j, k=1}^{n} \partial_{x_{j}}\left(a_{j k}(t, \cdot) \partial_{x_{k}} u\right)\right\|_{H^{-s}}^{2} d t \\
\quad \gtrsim \gamma^{1 / 4} \int_{0}^{T / 2} e^{\frac{2}{\gamma} \Phi(\gamma(T-t))}\left(\left\|\nabla_{x} u\right\|_{H_{\omega}^{-s}}^{2}+\gamma^{3 / 4}\|u\|_{L^{2}}^{2}\right) d t
\end{gathered}
$$

for all $u \in C_{0}^{\infty}\left(\mathbb{R}_{t} \times \mathbb{R}_{x}^{n}\right)$ with $\operatorname{supp}(u) \subseteq[0, T / 2] \times \mathbb{R}_{x}^{n}$ and all $\gamma \geq \gamma_{0}$.

The weight function in this Carleman estimate is the same as described in Remark 1.2 and the space $H_{\omega}^{-s}\left(\mathbb{R}_{x}^{n}\right)$ is defined by the Littlewood-Paley decomposition (see e.g. [12]):

$$
\|u\|_{H_{\omega}^{-s}}^{2}:=\sum_{\nu \geq 0} 2^{2(1-s) \nu} \omega^{2}\left(2^{-\nu}\right)\left\|\Delta_{\nu} u\right\|_{L^{2}}^{2}<+\infty .
$$

In this paper, we announce a generalization of the above mentioned results in $[5,6]$ to operators of type (1.1) with $m \geq 2$. The full proof, along with related results, will be published elsewhere [7]. The only existing result of this type, that the author is aware of, is [4], where the operator

$$
P=\partial_{t}+\sum_{0 \leq|\alpha| \leq 2 m} i^{|\alpha|} \partial_{x}^{\alpha}
$$


is considered. The precise statement there is

Proposition 1.5 (Prop. 2.1 in [4]). Let $\mu$ be a modulus of continuity satisfying (1.2) and $a_{\alpha} \in C^{\mu}[0, T]$. Then there exists a strictly increasing $C^{2}$-function $\Phi:[0,+\infty) \rightarrow[0,+\infty)$ such that there exists a $\gamma_{0} \geq 1$ such that

$$
\begin{gathered}
\int_{0}^{T / 2} e^{\frac{2}{\gamma} \Phi(\gamma(T-t))}\left\|\partial_{t} u-\sum_{0 \leq|\alpha| \leq 2 m} i^{|\alpha|} a_{\alpha}(t) \partial_{x}^{\alpha} u\right\|_{L^{2}}^{2} d t \\
\gtrsim \gamma^{\frac{1}{2}} \int_{0}^{T / 2} e^{\frac{2}{\gamma} \Phi(\gamma(T-t))}\|u\|_{H^{m}}^{2} d t
\end{gathered}
$$

for all $u \in C_{0}^{\infty}\left(\mathbb{R}_{t} \times \mathbb{R}_{x}^{n}\right)$ with $\operatorname{supp}(u) \subseteq[0, T / 2] \times \mathbb{R}_{x}^{n}$ and all $\gamma \geq \gamma_{0}$.

From the Carleman estimate follows that $P$ in (1.3) has the backward uniqueness property. The weight function is again the same as in Remark 1.2.

Our description of the history of the problem that we sketched here is by no means exhaustive. To get a better overview over the literature, the reader may consult the works referenced in the above cited works as well as in $[8,9]$. In [15], Tarama proved a similar result to the one in [5] but replacing the regularity measurement of the principal coefficients with respect to $t$ by a modulus of continuity by bounded variation. It would be interesting to see whether this results holds also for higher order operators.

Modifying a well known counterexample of Pliś [13], Del Santo and Prizzi proved in $[3,4]$ that the regularity assumptions with respect to $t$ in the above backward uniqueness results are essentially sharp. See Theorem 1.2 and Remark 1.2 in [4]. Up to now there are no counterexamples in the literature that involve $x$ in the principal part to show the sharpness of the assumptions with respect to $x$.

Notation. By $B^{2}\left(\mathbb{R}_{x}^{n}\right)$, we denote the twice differentiable functions on $\mathbb{R}_{x}^{n}$ which are bounded with all derivatives of order $\leq 2$. Given a modulus of continuity $\mu, C^{\mu}[0, T]$ denotes the space of continuous functions $f$ that satisfy $|f(s)-f(t)| \leq C \mu(|t-s|)$. The space $W^{k, \infty}\left(\mathbb{R}_{x}^{n}\right)$ denotes the space of all functions $f \in L^{\infty}\left(\mathbb{R}_{x}^{n}\right)$ such that all distributional derivatives $\partial_{x}^{\gamma} u \in L^{\infty}\left(\mathbb{R}_{x}^{n}\right)$ for $|\gamma| \leq k$.

\section{The main result}

The main result of this paper is

Theorem 2.1 (Uniqueness). Consider the operator $P$, defined by the equation

$$
P u=\partial_{t} u+\sum_{|\alpha|,|\beta|=m}(-1)^{|\alpha|} \partial_{x}^{\alpha}\left(a_{\alpha \beta}(t, x) \partial_{x}^{\beta} u\right)+\sum_{|\gamma| \leq m} b_{\gamma}(t, x) \partial_{x}^{\gamma}=0
$$

with real $a_{\alpha \beta}$ and possibly complex $b_{\gamma}$. We assume that

- $a_{\alpha \beta} \in C^{\mu}\left([0, T], L^{\infty}\left(\mathbb{R}_{x}^{n}\right)\right) \cap L^{\infty}\left([0, T], W^{m, \infty}\left(\mathbb{R}_{x}^{n}\right)\right)$, 
- $b_{\gamma} \in L^{\infty}\left([0, T] \times \mathbb{R}_{x}^{n}\right)$,

- there exists a $\kappa \in(0,1]$ such that $\sum_{|\alpha|=|\beta|=m} a_{\alpha \beta}(t, x) \xi^{\alpha} \xi^{\beta} \geq \kappa|\xi|^{2 m}$ for all $(t, x, \xi) \in[0, T] \times \mathbb{R}_{x}^{n} \times \mathbb{R}_{\xi}^{n}$.

Then $P$ has the backward uniqueness property in $\mathcal{H}^{m}$, i.e. for $u \in \mathcal{H}^{m}, P u=$ 0 on $[0, T] \times \mathbb{R}_{x}^{n}$ and $u(T, x)=0$ on $\mathbb{R}_{x}^{n}$ it follows $u=0$ on $[0, T] \times \mathbb{R}_{x}^{n}$.

This result is an extension of [4] generalizing [5] to higher order operators. As in the other cases it follows in a standard way from an appropriate Carleman estimate.

Proposition 2.2 (Carleman estimate). Let $\mu$ be a modulus of continuity satisfying (1.2). There exists a strictly increasing $C^{2}$-function $\Phi:[0,+\infty) \rightarrow$ $[0,+\infty)$ and $a \gamma_{0} \geq 1$ such that

$$
\begin{aligned}
& \int_{0}^{T / 2} e^{\frac{2}{\gamma} \Phi(\gamma(T-t))}\left\|\partial_{t} u-\sum_{|\alpha|,|\beta|=m}(-1)^{m} \partial_{x}^{\alpha}\left(a_{\alpha \beta}(t, \cdot) \partial_{x}^{\beta} u\right)\right\|_{L^{2}}^{2} d t \\
& \quad \gtrsim \gamma^{\frac{1}{2}} \int_{0}^{T / 2} e^{\frac{2}{\gamma} \Phi(\gamma(T-t))}\|u\|_{H^{m}}^{2} d t
\end{aligned}
$$

for all $u \in C_{0}^{\infty}\left(\mathbb{R}_{t} \times \mathbb{R}_{x}^{n}\right)$ with $\operatorname{supp}(u) \subseteq[0, T / 2] \times \mathbb{R}_{x}^{n}$ and all $\gamma \geq \gamma_{0}$.

Remark 2.3. The proof of this result is an extension of the proofs in $[5,6]$ with weight function $\Phi$ from Remark 1.2. To treat different lower order terms in (2.1), especially terms of the form $\sum_{|\gamma|=2 m-1} b_{\gamma}(t, x) \partial_{x}^{\gamma} u$, new ideas are required. This problem will be discussed in a forthcoming paper [7]. Contrary to Proposition 1.5, it is reasonable to expect that the regularity assumption with respect to $t$ can be lowered for the terms of order $\leq 2 m-1$.

To prove this Carleman estimate, one follows the strategy of $[5,6,4]$. The full proof as well as an extension in the spirit of [6] will appear in a forthcoming paper [7]. Here we line out the main steps of the proof of (2.2):

1. We replace $v(t, x)=e^{\frac{1}{\gamma} \Phi(\gamma(T-t))} u(t, x)$ and rewrite $(2.1)$ in terms of $v$ :

$$
\begin{aligned}
\int_{0}^{T / 2} & \left\|\partial_{t} v-\sum_{|\alpha|,|\beta|=m}(-1)^{m} \partial_{x}^{\alpha}\left(a_{\alpha \beta}(t, \cdot) \partial_{x}^{\beta} v\right)+\Phi^{\prime}(\gamma(T-t)) v\right\|_{L^{2}}^{2} d t \\
& \gtrsim \gamma^{\frac{1}{2}} \int_{0}^{T / 2}\|v\|_{H^{m}}^{2} d t .
\end{aligned}
$$

2. In this step we use Bony's paraproduct as introduced in [2]. The operator is defined as $T_{a} u=\sum_{\nu>N} S_{\nu-N} a \Delta_{\nu} u$, where $S_{\nu}$ is an operator localizing to $\left\{|\xi| \leq 2^{\nu+1}\right\}$ in the phase space and $\Delta_{\nu}$ is localizing to $\left\{2^{\nu-1} \leq|\xi| \leq 2^{\nu+1}\right\}$ in the phase space. For more information and the properties of $T_{a}$, we refer to Bony's paper [2] and [5, 6, 9]. We replace $a_{\alpha \beta}$ by $T_{a_{\alpha \beta}}$ and use the fact that $a_{\alpha \beta}-T_{a_{\alpha \beta}}$ is $m$-regularizing for 


$$
\begin{aligned}
& a_{\alpha \beta} \in W^{m, \infty} \text {. Thus, the analysis can be reduced to proving } \\
& \int_{0}^{T / 2}\left\|\partial_{t} v-\sum_{|\alpha|,|\beta|=m}(-1)^{m} \partial_{x}^{\alpha}\left(T_{a_{\alpha \beta}} \partial_{x}^{\beta} v\right)+\Phi^{\prime}(\gamma(T-t)) v\right\|_{L^{2}}^{2} d t \\
& \quad \gtrsim \gamma^{\frac{1}{2}} \int_{0}^{T / 2}\|v\|_{H^{m}}^{2} d t .
\end{aligned}
$$

3. We microlocalize (2.3) by writing the norms in terms of LittlewoodPaley decompositions. Using appropriate estimates (similar to the estimates in $[5,6])$ for

$$
\sum_{|\alpha|,|\beta|=m}(-1)^{m} \partial_{x}^{\alpha}\left[\Delta_{\nu}, T_{a_{\alpha \beta}}\right] \partial_{x}^{\beta} u
$$

the analysis is reduced to a term by term analysis of

$$
\int_{0}^{T / 2}\left\|\partial_{t} v_{\nu}-\sum_{|\alpha|,|\beta|=m}(-1)^{m} \partial_{x}^{\alpha}\left(T_{a_{\alpha \beta}} \partial_{x}^{\beta} v_{\nu}\right)+\Phi^{\prime}(\gamma(T-t)) v_{\nu}\right\|_{L^{2}}^{2} d t .
$$

4. The proof proceeds in performing integration by parts with respect to $t$ on the term

$$
2 \operatorname{Re} \int_{0}^{T / 2}\left\langle\partial_{t} v_{\nu} \mid-\sum_{|\alpha|,|\beta|=m}(-1)^{m} \partial_{x}^{\alpha}\left(T_{a_{\alpha \beta}} \partial_{x}^{\beta} v_{\nu}\right)\right\rangle d t
$$

Due to the low-regularity of $a_{\alpha \beta}$ with respect to $t$, we have to regularize the coefficients. We use a standard mollifying technique and use estimates for $T_{\partial_{t} a_{\alpha \beta}^{\epsilon}}$ and $T_{a_{\alpha \beta}-a_{\alpha \beta}^{\epsilon}}$. These are the same as in $[3,5,6]$.

5. We will estimate the microlocalized pieces term by term and handle low and high frequencies separately to obtain (2.3) by summing up all pieces.

\section{References}

[1] C. Bardos and L. Tartar Sur l'unicité rétrograde des équations paraboliques et quelques questions voisines, Archive for Rational Mechanics and Analysis, 50(1) (1973), pp. 10-25

[2] J.-M. Bony Calcul symbolique et propagation des singularités pour les équations aux dérivées partielles non linéaires, Annales scientifiques de l'École Normale Supérieure, 14(2) (1981), pp. 209-246

[3] D. Del Santo and M. Prizzi Backward uniqueness for parabolic operators whose coefficients are non-Lipschitz continuous in time, Journal de Mathématiques Pures et Appliquées, 84(4) (2005), pp. 471-491.

[4] D. Del Santo and M. Prizzi On the backward uniqueness property for a class of parabolic operators in Phase Space Analysis of Partial Differential Equations, Progress in Nonlinear Differential Equations and Their Applications, 69 (2007), Birkhäuser, pp. 95-105.

[5] D. Del Santo and M. Prizzi A new result on backward uniqueness for parabolic operators, Annali di Matematica Pura ed Applicata, 194(2) (2015), pp. 387-403. 
[6] D. Del Santo and Ch. P. Jäh and M. Paicu Backward-uniqueness for parabolic operators with non-Lipschitz coefficients, Osaka Journal of Mathematics, 52(3) (2015), pp. 793-817.

[7] Ch. P. Jäh Backward uniqueness for a class of higher order parabolic equations with low-regular coefficients, in preparation.

[8] Ch. P. Jäh, Uniqueness and Non-uniqueness in the Cauchy Problem for Elliptic and Backward-Parabolic Operators, Diploma Thesis, Technische Universität Bergakademie Freiberg, 2011

[9] Ch. P. Jäh Some results on the uniqueness and conditional stability in the Cauchy problem for backward-parabolic equations with low-regular coefficients, PhD Thesis, Technische Universität Bergakademie Freiberg, 2015.

[10] K. Miller Nonunique continuation for uniformly parabolic and elliptic equations in self-adjoint divergence form with Hölder continuous coefficients, Archive for Rational Mechanics and Analysis, 54(2) (1973), pp. 105-117.

[11] J.-L. Lions and B. Malgrange Sur l'unicité rétrograde dans les problèmes mixtes paraboliques, Mathematica Scandinavica, 8 (1960), pp. 277-286.

[12] G. Métivier Para-differential Calculus and Applications to the Cauchy Problem for Nonlinear Systems, Centro di Ricerca Matematica Ennio De Giorgi (CRM) Series, 5. Edizioni della Normale, Pisa, 2008.

[13] A. Pliś On non-uniqueness in Cauchy problem for an elliptic second order differential equation, Bulletin de l'Académie Polonaise des Sciences, Série des Sciences Mathématiques, 11 (1963), pp. 95-100.

[14] S. Tarama Local uniqueness in the Cauchy problem for second order elliptic equations with non-Lipschitzian coefficients, Publications of the Research Institute for Mathematical Sciences - Kyoto University, 33 (1997), pp. 167-188.

[15] S. Tarama Backward uniqueness for heat equations with coefficients of bounded variation in time, Electronic Journal of Differential Equations 189 (2013), pp. $1-14$.

Christian P. Jäh

Department of Mathematical Sciences

Loughborough University

Loughborough, Leicestershire, LE11 3TU

United Kingdom

e-mail: c.jaeh@lboro.ac.uk 